\title{
Valores típicos de dosis para tomografía computada de cerebro en pacientes adultos
}

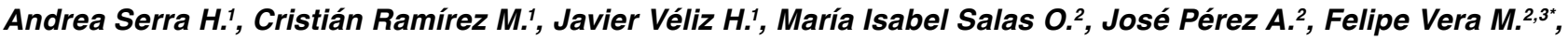 \\ Juan Morales G. ${ }^{2,4}$ \\ 1. Estudiante Tecnología Médica. Escuela Tecnología Médica, Facultad Medicina, Universidad de Valparaíso, Valparaíso, \\ Chile. \\ 2. Tecnólogo Médico Imagenología y Física Médica. Escuela Tecnología Médica, Facultad Medicina, Universidad de \\ Valparaíso, Chile. Servicio Imagenología, Clínica BUPA Reñaca, Viña del Mar, Chile. \\ 3. Magíster en administración con especialización en salud. Escuela Tecnología Médica, Facultad Medicina, Universidad \\ de Valparaíso, Chile. Servicio Imagenología, Clínica Bupa Reñaca, Viña del Mar, Chile. \\ 4. Profesor auxiliar Escuela Tecnología Médica, Facultad Medicina, Universidad de Valparaíso, Valparaíso, Chile.
}

\section{Typical dose values for brain computed tomography in adult patients}

Resumen: Debido al aumento en el uso de la Tomografía Computada (TC), y en consecuencia, la probabilidad de generar un incremento progresivo de la dosis recibida por los paciente y su relación en el potencial riesgo de los efectos de las radiaciones ionizantes, es importante implementar el uso de niveles de referencia diagnóstico (DRLs) en TC, como herramienta fundamental dentro de un programa de control de calidad que permita la evaluación y optimización de las dosis entregadas a los pacientes según la tarea clínica deseada. Con el objetivo de establecer valores típicos de dosis en TC de cerebro en Clínica Bupa Reñaca, se estudió una muestra de 73 informes dosimétricos generados en un equipo TC Toshiba Aquilion 64, en términos de indicadores de dosis para TC: Índice de dosis en TC por volumen (CTDIvol) y Producto dosis longitud (DLP). Con los datos obtenidos, se estimó el valor del percentil 50 (p50) para cada indicador de dosis, y se determinaron los valores típicos de dosis en cada grupo estudiado según sexo, edad e indicación clínica. Se logró definir y establecer una metodología que permitió la obtención de los valores típicos de dosis para TC de cerebro, optimizando las dosis sin producir una disminución en la calidad de la imagen necesaria para nuestro propósito clínico.

Palabras clave: Dosis de radiación, Niveles diagnósticos de referencia, Optimización de la dosis, Tomografía computada de cerebro, Valor típico de dosis.

Abstract: Due to the increase in the use of Computed Tomography (CT), and consequently, the probability of generating a progressive increase in the dose received by the patient and its relationship in the potential risk of the effects of ionizing radiation, it is important to implement the use of diagnostic reference levels (DRLs) in CT, as a fundamental tool within a quality control program that allows the evaluation and optimization of the doses delivered to patients according to the desired clinical task.

In order to establish typical dose values in brain CT at Bupa Reñaca Clinic, a sample of 73 dosimetric reports generated on a Toshiba Aquilion 64 CT unit was studied, in terms of dose indicators for CT: Dose rate on CT by volume (CTDIvol) and Dose Length Product (DLP). With the data obtained, the value of the 50 th percentile ( $p 50)$ for each dose indicator was estimated, and typical dose values were determined in each group studied according to sex, age and clinical indication. It was possible to define and establish a methodology that allowed obtaining typical dose values for brain CT, optimizing the doses without producing a decrease in the image quality necessary for our clinical purpose.

Keywords: Brain computed tomography, Diagnostic reference levels, Optimization of the dose, Radiation dose, Typical dose value.

Serra A, et al. Valores típicos de dosis para tomografía computada de cerebro en pacientes adultos. Rev Chil Radiol 2020; 26(1): 25-31.

*Correo electrónico: Felipe Vera Muñoz / f_vera85@yahoo.es.

Trabajo enviado el 30 de septiembre de 2019. Aceptado para publicación el 17 de marzo de 2020.

Fuente de apoyo financiero: Protección Radiológica Ltda - Eduardo Espinoza Parra

Influencia en diseño del estudio: Sí

Clínica Bupa Reñaca - Servicio de Imagenología

Influencia en diseño del estudio: No 


\section{Introducción}

El uso de la radiación ionizante en el área de la medicina es una práctica que evoluciona rápidamente. Según el Comité Científico de Naciones Unidas sobre los Efectos de la Radiación Atómica (UNSCEAR), lo anterior se debe en gran parte a la introducción de nuevas técnicas de imagen como la tomografía computada (TC) Multidetector, imagen digital y al alto nivel de innovación de las empresas fabricantes de equipos. De igual forma, el acortamiento de los tiempos de exploración, junto con un rápido proceso de reconstrucción de imágenes gracias a la informática actual, ha determinado una mayor demanda de estudios bajo esta técnica. UNSCEAR, reportó en el año 2010 que las TC contribuyen con el 43\% de la dosis colectiva mundial correspondiente a radiología médica diagnóstica. Como consecuencia, el aumento en la frecuencia de este examen tiene un impacto significativo dentro de la dosis total de la población producto de exposiciones médicas ${ }^{(1)}$.

Se debe considerar que la TC se ha convertido en una técnica vital para el estudio de enfermedades, su estadificación y su control. Las imágenes obtenidas son de gran calidad y representan fielmente las distintas estructuras y órganos contenidos en el barrido, por lo que actualmente se considera como una herramienta trascendental a la hora de diagnosticar, estadificar, controlar y planificar el tratamiento de ciertas patologías; además de servir como guía en procedimientos invasivos, toma de biopsias y drenaje de abscesos ${ }^{(2)}$. Sin embargo, es necesario recordar que el uso de radiación ionizante está asociado a un riesgo probable de desencadenar efectos biológicos adversos y posibles daños a la salud del paciente. En su mayoría, las patologías asociadas secundarias a la exposición por radiación podrían disminuirse con una adecuada intervención preventiva y protectora(3).

Para responder a estas problemáticas, la Comisión Internacional de Protección Radiológica (ICRP) introdujo en la década de los noventa, el concepto de Niveles de Referencia Diagnóstico (DRLs) en TC(4). Dentro de ello, publicaciones actuales basadas en las recomendaciones de la ICRP señalan que se deben adoptar medidas de protección radiológica a fin de prevenir, en lo posible, dosis innecesariamente altas durante las exposiciones médicas a los pacientes ${ }^{(5)}$. De acuerdo a las recomendaciones de la ICRP, el énfasis se debe poner en la justificación de los exámenes radiológicos, en la optimización de la protección y en la utilización de los niveles de referencia para diagnóstico(6).

En Chile, según las cifras expuestas por el Departamento de Estadísticas e Información en Salud (DEIS $^{(7,8)}$ durante el año 2018 se realizaron 1.136 .703 exámenes diagnósticos por TC a nivel nacional, de los cuales 113.563 corresponden al total de exámenes registrados por el DEIS en la Región de Valparaíso; estos datos revelan un incremento del 8,7\% a nivel país y un aumento del $12 \%$ a nivel regional en comparación con los registros del año 2017 de la misma entidad $(9,10)$. De los datos revelados por el DEIS, es importante agregar que las TC realizadas en establecimientos no pertenecientes al Sistema Nacional de Servicios de Salud, registraron un total de 727.562 exámenes, que sumados al 1.037.787 de TC realizados en el sector público, corresponden a un $41,2 \%$ de los TC totales registrados en el país durante el año 2017, reflejando que las instituciones privadas aportan un número considerable de las exploraciones tomográficas, lo que representa un dato relevante en la elección de la institución para este estudio(11).

Los DRLs son una herramienta utilizada para optimizar la protección radiológica en la exposición médica de pacientes en procedimientos diagnósticos e intervencionales, facilitando el logro de un rango de distribución homogéneo, más estrecho y óptimo de la dosis de radiación para un examen radiológico (de requisitos técnicos y clínicos específicos), por lo que su uso, revisión y acciones derivadas, según corresponda, ayudarían a optimizar los protocolos de estudio ${ }^{(12,6)}$, esto sin afectar la calidad de imagen adquirida. Como condición deben cuantificarse en términos fácilmente reproducibles, a modo de simplificar su uso en la práctica clínica habitual.

Las magnitudes dosimétricas recomendadas en TC son los indicadores de dosis, derivados del Índice de dosis (CTDI): Índice de dosis en tomografía computarizada ponderado (CTDlw) e Índice de dosis en tomografía computarizada volumétrica (CTDlvol) respectivamente, más el Producto Dosis Longitud $(\mathrm{DLP})^{(6)}$. Estos indicadores pueden ser obtenidos directamente en los reportes dosimétricos de cada estudio de TC, lo que facilita el nivel de registro y el establecimiento de un valor típico de dosis para un determinado estudio, como medida inicial a fin de lograr a nivel local la creación de DRLs.

Entidades regionales internacionales, como la Directiva sobre Exposiciones Médicas de la Comisión Europea han fijado políticas y normativas para promover la utilización y establecimiento de estos niveles en la práctica radiológica habitual, siguiendo así las recomendaciones de la Agencia Internacional de Energía Atómica $(I A E A)^{(6,11)}$. A nivel nacional, si bien el Instituto de Salud Pública (ISP) reconoce la importancia de aplicar DRLs, actualmente en Chile no existe un marco legal, ni un soporte bien establecido para desarrollar DRLs en TC, ni en otra modalidad de radiodiagnóstico ${ }^{(13)}$. Lo anterior, explica la razón por la cual no existen directrices ni entes reguladores específicos en esta materia, y el por qué la mayor parte de los profesionales implicados en el área declaran desconocer estos términos. Sin embargo, en el corto plazo esta situación podría cambiar gracias a un nuevo reglamento de protección radiológica que se 
encuentra en proceso de aprobación legislativa para Chile, el cual servirá de base para actualizaciones futuras $^{(13,14,15)}$.

La investigación pretende acercar el uso de los valores típicos de dosis a un centro en particular; orientando a la comunidad radiológica implicada, y junto con ello, mediante los resultados obtenidos, contribuir a complementar los estudios existentes a nivel nacional respecto a esta temática a fin de facilitar la planificación, preparación e implementación de las posibles normativas venideras, optimizando los protocolos de estudio bajo las recomendaciones de entidades de referencia como ICRP(6). Los resultados obtenidos a partir de este estudio constituirán el primer paso para la optimización de la dosis en pacientes que se realicen TC de cerebro con protocolo de estudio estándar basado en la metodología propuesta; cuya consecución de proceso llevará a establecer un valor típico de dosis en el departamento de Imagenología de Clínica Bupa Reñaca.

\section{Material y método}

Este estudio fue realizado de forma observacional, transversal y descriptiva entre los meses de junio 2017 a enero del año 2018, tomando como muestra los reportes estructurados de dosis de radiación (RSDR), en forma secuencial, de los exámenes de TC de cerebro sin contraste realizados en la Unidad de Imagenología de Clínica Bupa Reñaca - Viña del Mar. Los reportes se utilizaron específicamente para la obtención de los parámetros de dosis: CTDIvol y DLP. A partir de la recolección de datos secuenciales, la muestra se dirigió de forma tal que cubriera al menos 30 pacientes ${ }^{(16)}$, esto para cubrir el rango intercuartil, el cual sirve como un indicador de la dispersión de datos $^{(12)}$. La información obtenida de los pacientes no incluye datos personales sino solo sus parámetros dosimétricos, por lo que el estudio fue autorizado por la dirección del Servicio sobre estos términos de confidencialidad de los datos resultantes.

Los exámenes fueron adquiridos con un tomógrafo computado marca Toshiba, modelo Aquilion 64 TSX - 101A, año de fabricación 2007, kilovolt peak $(\mathrm{kVp})$ máximo 135 y miliamperio $(\mathrm{mA})$ máximo 500. Los parámetros de adquisición del estudio fueron: Modalidad Helicoidal, voltaje tubo $120 \mathrm{kVp}$, corriente $250 \mathrm{~mA}$, tiempo rotación tubo 0.75 segundos, pitch 0.844, D-FOV 240.0 (small), configuración de matriz adquisición $0.5 \times 32$, dirección de barrido caudo - craneal, reconstruido mediante retroproyección filtrada (método analítico). Con lo anterior, se recolectaron los elementos primarios del estudio, es decir los informes dosimétricos generados automáticamente por cada examen de TC de cerebro con dicho protocolo estándar que incluían CTDIvol y DLP calculados mediante algoritmos matemáticos estandarizados.

Para certificar que los datos con los que se tra- bajó eran reproducibles, se realizó una evaluación dosimétrica previa del tomógrafo a fin de establecer una correlación entre los valores entregados por el equipo con los valores medidos y registrados. Dichas mediciones se efectuaron conforme a las bases del protocolo IAEA human health series n.19 (test anual) y las referencias de la sociedad ImPACTScan ${ }^{(17)}$. EI procedimiento contempló la medición de la dosis real implicada en la exposición, la cual fue captada por una cámara de ionización tipo lápiz (Radcal 10X6-3CT, de $10 \mathrm{~cm}$ de largo y $3 \mathrm{~cm}^{3}$ de capacidad) en un fantoma de cabeza cilíndrico de polimetilmetacrilato (PMMA) de $16 \mathrm{~cm}$ de diámetro; sus lecturas fueron obtenidas a partir del módulo digitalizador Radcal AGDM+ y su conexión USB al software Accu-Gold. La prueba contempló un nivel de aceptación de +/- 20\%(18); por lo que se comprobó que los valores dictados por la consola se encontraban dentro del rango estipulado, y que se corresponden con la dosis medida con la cámara de ionización. Debido a que los valores de CTDIvol y DLP nominales de la consola son adquiridos a partir de fórmulas computacionales estandarizadas según las dimensiones del fantoma, se asegura el cumplimiento de esta prueba para todas las modalidades, factores de exposición y colimación aplicables a protocolos calibrados mediante un fantoma cilíndrico de $16 \mathrm{~cm}$ de diámetro. A lo anterior, se debe sumar que el equipo utilizado para realizar la experiencia cuenta con un plan de mantenimiento trimestral directo con el proveedor. Ambos antecedentes buscan dejar en claro que el equipamiento utilizado se encuentra dentro de un marco de control y garantía de calidad.

La investigación se ejecutó a partir de los siguientes datos: indicadores de dosis en TC (CTDIvol y DLP), diagnóstico o indicación clínica y los datos demográficos como sexo y edad de los pacientes. Cada subgrupo se conformó por diez o más elementos (reportes de dosis), cumpliendo con los parámetros propuestos por la IAEA para asegurar que la dosis a cada paciente sea tan baja como sea razonablemente posible, conforme al objetivo clínico del examen ${ }^{(4)}$, a fin de establecer valores típicos de dosis. El grupo total del estudio fue de 73 reportes dosimétricos, superando los requerimientos básicos estipulados por la ICRP ${ }^{(12)}$ para determinar valores típicos de dosis en la modalidad de TC. Si bien, en estudios de cerebro, la estandarización de la estructura es sencilla debido a que sigue dimensiones recurrentes, siempre se debe tener en cuenta el tamaño o dimensiones de la estructura objeto que se desee analizar dado que los parámetros técnicos a utilizar varían en función de esta última variable.

Mediante el software XLSTAT con función estadísticos descriptivos, se estimaron los valores mínimo y máximo, percentil 50 , media y desviación estándar (SD) de las variables CTDIvol y DLP, para cada subgrupo demográfico de sexo (hombre o mujer) y edad (18 a 
45 años y mayores de 45 años), así como de indicación clínica (ACV, TEC o cefalea) y para la totalidad de la muestra. Además, se determinó los intervalos de confianza (IC) correspondientes a los estimadores mediana (p50) de cada subgrupo de sexo y edad, y de la globalidad de los datos utilizando la técnica Bootstrap: función que toma múltiples muestras con reemplazo a partir de la muestra original, calculando los estimadores requeridos de cada muestra obtenida y promediándolos para obtener valores aplicables a la población en estudio. Finalmente, los datos se presentan en tablas comparativas.

\section{Resultados}

Los resultados fueron obtenidos en función del valor p50 y en términos de indicadores de dosis en TC (CTDIvol y DLP), además de otros estimadores estadísticos correspondientes a los exámenes de pacientes agrupados por sexo, edad e indicación clínica. Los valores estimados se analizaron con un nivel de confianza del $95 \%$, y en el cual se encontraron todas las variables.

Para los valores típicos de dosis por sexo, el p50, además de otros estimadores estadísticos se representan en la tabla 1. Los valores típicos de dosis para CTDIvol y DLP obtenidos en los pacientes según sexo no representan significancia estadística.

Para los valores típicos de dosis especificados según la variable edad, la muestra fue subdividida en dos grupos etarios: pacientes entre 18 a 45 años y mayores de 45 años, donde se obtuvo un valor p50 en CTDlvol y DLP junto con el resto de parámetros estadísticos. Los resultados de la tabla 2 evidencian valores típicos sin diferencia estadísticamente significativa.

Para los valores típicos de dosis según la indicación clínica de la TC de cerebro, la muestra se subdividió en cinco indicaciones de realización del examen: Traumatismo encéfalo craneal (TEC), accidente cerebro vascular (ACV), cefalea, pérdida de conciencia y otros. En la tabla 3 se demuestra el valor típico de dosis agrupado para cada patología en valores de p50 y los restantes estimadores estadísticos. En cuanto a los valores típicos obtenidos no representan significancia estadística.

Finalmente, los valores p50 además de los otros estimadores estadísticos correspondientes a la totalidad de exámenes de TC de cerebro evaluados, se detallan en la tabla 4. Por consiguiente, los valores típicos de dosis globales obtenidos para los indicadores CTDIvol y DLP son $52.4 \mathrm{mGy}$ y $1087.4 \mathrm{mGy}^{*} \mathrm{~cm}$.

\section{Discusión}

De acuerdo al análisis de la muestra estudiada, se encontró que los valores CTDIvol y DLP obtenidos en el total de los exámenes realizados corresponden a los valores típicos de dosis de la institución para una TC de cerebro en pacientes adultos. Se utilizó el concepto de "valores típicos", debido a que se trata de un centro donde existe sólo un equipo de TC, siendo demasiado pequeño para la determinación de un valor DRL Local, no obstante, es interesante establecer un criterio razonable que permita dimensionar las diferencias entre lo propuesto y lo obtenido en términos de valores a nivel institucional, local o nacional ${ }^{(12)}$. Sin embargo, fue posible construir una metodología fácilmente reproducible en cuanto al desarrollo de la experiencia y análisis estadístico, permitiendo desarrollar una estrategia de optimización de las dosis, todo dentro de un marco de control y garantía de calidad diagnóstica.

Tal y como lo han manifestado otros autores ${ }^{(19)} \mathrm{el}$ problema fundamental sobre la dosis entregada a los pacientes en el área de TC ha sido la integración casi

Tabla 1. Valores típicos de dosis (p50) y estimaciones estadísticas en términos de CTDlvol y DLP de las tomografías computadas de cerebro en pacientes adultos según sexo.

\begin{tabular}{|c|c|c|c|c|}
\hline Estimadores & CTDIvol (mGy) & $\mathrm{DLP}\left(\mathrm{mG} \mathbf{y}^{*} \mathrm{~cm}\right)$ & CTDIvol (mGy) & $\mathrm{DLP}\left(\mathrm{mG} \mathrm{y}^{*} \mathrm{~cm}\right)$ \\
\hline Número observaciones & \multicolumn{2}{|c|}{$\begin{array}{c}\text { Femenino } \\
38\end{array}$} & \multicolumn{2}{|c|}{ Masculino } \\
\hline Valor mínimo & 50,100 & 961,2 & 50,100 & 966,6 \\
\hline Valor máximo & 80,200 & 1459,2 & 66,800 & 1316,2 \\
\hline Mediana (p50) & 50,100 & 1084,5 & 52,400 & 1110,7 \\
\hline Media & 52,679 & 1097,758 & 52,260 & 1108,263 \\
\hline Desviación estándar & 5,906 & 93,055 & 3,812 & 76,911 \\
\hline
\end{tabular}


Tabla 2. Valores típicos de dosis (p50) y estimaciones estadísticas en términos de CTDIvol y DLP de las tomografías computadas de cerebro según rango de edad.

\begin{tabular}{|c|c|c|c|c|}
\hline Estimadores & CTDIvol (mGy) & DLP $\left(m G y^{*} \mathrm{~cm}\right)$ & CTDIvol (mGy) & $\mathrm{DLP}\left(\mathrm{mG}^{*} \mathrm{~cm}\right)$ \\
\hline Número observaciones & \multicolumn{2}{|c|}{$\begin{array}{c}18 \text { a } 45 \text { años } \\
25\end{array}$} & \multicolumn{2}{|c|}{ Mayores 45 años } \\
\hline Valor mínimo & 50,1 & 961,2 & 50,100 & 966,600 \\
\hline Valor máximo & 52,4 & 1215,5 & 80,200 & 1459,200 \\
\hline Mediana (p50) & 50,1 & 1084,5 & 52,400 & 1087,400 \\
\hline Media & 51,112 & 1087,26 & 53,190 & 1110,885 \\
\hline Desviación estándar & 1,165 & 65,825 & 5,986 & 93,439 \\
\hline
\end{tabular}

Tabla 3. Valores típicos de dosis (p50) y estimaciones estadísticas en términos de CTDIvol y DLP de las tomografías computadas de cerebro según indicación clínica.

\begin{tabular}{|c|c|c|c|c|c|c|c|c|c|c|}
\hline Estimadores & $\begin{array}{l}\text { CTDIvol } \\
\text { (mGy) }\end{array}$ & $\begin{array}{c}\text { DLP } \\
\left(m G y^{*} \mathbf{c m}\right)\end{array}$ & $\begin{array}{c}\text { CTDIvol } \\
\text { (mGy) }\end{array}$ & $\begin{array}{c}\text { DLP } \\
\left(m G y^{*} \mathbf{c m}\right)\end{array}$ & $\begin{array}{l}\text { CTDIvol } \\
\text { (mGy) }\end{array}$ & $\begin{array}{c}\text { DLP } \\
\left(m G y^{*} \mathbf{c m}\right)\end{array}$ & $\begin{array}{c}\text { CTDIvol } \\
\text { (mGy) }\end{array}$ & $\begin{array}{c}\text { DLP } \\
\left(m G y^{*} \mathrm{~cm}\right)\end{array}$ & $\begin{array}{l}\text { CTDIvol } \\
\text { (mGy) }\end{array}$ & $\begin{array}{c}\text { DLP } \\
\left(\mathrm{mGy}{ }^{*} \mathrm{~cm}\right)\end{array}$ \\
\hline & \multicolumn{2}{|c|}{ Cefalea } & \multicolumn{2}{|c|}{ ACV } & \multicolumn{2}{|c|}{ TEC } & \multicolumn{2}{|c|}{ ompromiso conciencia } & \multicolumn{2}{|c|}{ Otros } \\
\hline $\mathrm{N}^{\circ}$ observaciones & \multicolumn{2}{|c|}{19} & \multicolumn{2}{|c|}{16} & \multicolumn{2}{|c|}{11} & \multicolumn{2}{|c|}{10} & \multicolumn{2}{|c|}{17} \\
\hline Valor mínimo & 50,1 & 987,1 & 50,1 & 987,1 & 50,1 & 961,2 & 50,1 & 966,6 & 50,1 & 1032,1 \\
\hline Valor máximo & 66,8 & 1282,8 & 66,8 & 1316,2 & 52,4 & 1189,3 & 52,4 & 1215,5 & 80,2 & 1459,2 \\
\hline Mediana (p50) & 50,1 & 1087,4 & 52,4 & 1110,7 & 52,4 & 1078,7 & 50,1 & 1037,2 & 52,4 & 1112,4 \\
\hline Media & 51,705 & 1091,316 & 53,625 & 1111,631 & 51,355 & 1083,555 & 51,02 & 1072,42 & 53,847 & 1137,624 \\
\hline Desviación estándar & 3,813 & 76,219 & 5,24 & 84,023 & 1,201 & 65,292 & 1,188 & 73,159 & 7,856 & 107,407 \\
\hline
\end{tabular}

Tabla 4. Valores típicos de dosis (p50) y estimaciones estadísticas en términos de CTDIvol y DLP del total de tomografías computadas de cerebro evaluadas.

\begin{tabular}{|lcc|}
\hline Estimadores & CTDIvol (mGy) & DLP (mGy $\left.{ }^{*} \mathbf{c m}\right)$ \\
\hline & & \\
Número observaciones & 73 & \\
Valor mínimo & 50,1 & 961,2 \\
Valor máximo & 80,2 & 1459,2 \\
Mediana (p50) & $\mathbf{5 2 , 4}$ & $\mathbf{1 0 8 7 , 4}$ \\
Intervalo confianza (p5) & $\mathbf{5 0 , 1 - 5 2 , 4}$ & $\mathbf{1 0 6 2 , 3 - 1 1 1 2 , 4}$ \\
Media & 52,478 & 1102,795 \\
Desviación estándar & 4,983 & 85,271 \\
\hline
\end{tabular}


exclusiva del CTDI y sus derivados: CTDIvol y DLP. Independiente de la innegable contribución que han representado estos conceptos, facilitando la implementación de políticas regulatorias, aplicaciones clínicas y consenso dosimétrico entre fabricantes de $\mathrm{TC}^{(20,21)}$ estandarizando de forma considerable las medidas de este valor, pero con el inconveniente de no poder ser consideradas como un indicador de dosis entregada al paciente ${ }^{(19)}$, principalmente porque el CTDI no toma en consideración el tamaño del paciente. Si bien el CTDlvol se mide en el equipo mediante fantomas cilíndricos de PMMA, homogéneos y de diferentes diámetros, como por ejemplo en fantoma craneal donde presenta un tamaño estándar de $16 \mathrm{~cm}$, no es totalmente representativo de la anatomía real de cualquier paciente, y si bien, aunque nos ayuda a obtener una referencia de dosis, en ningún caso podemos aplicarlos para representar la dosis efectiva a un determinado paciente. Dado lo anterior, el desarrollo de la metodología propuesta y la generación de valores típicos de dosis permitieron utilizar esta información a fin de generar una referencia institucional que facilite y contribuya a la ejecución de una futura iniciativa local, regional o nacional en función de una estructura anatómica fácilmente estandarizable sobre todo en lo que respecta a sus dimensiones.

Si bien para el análisis dosimétrico estructural se recomienda no separar las variables estudiadas dada la baja variabilidad existente entre los criterios de inclusión de la investigación, los autores decidieron aperturar estos a fin de comprobar, si existía o no cambios en el comportamiento de la información. Obteniendo como resultados nulas diferencias estadísticas entre las variables analizadas.

Sin embargo, en el estudio se debe hacer especial énfasis en los valores dosimétricos obtenidos en función del DLP, principalmente por el método de adquisión del protocolo seleccionado. En la modalidad helicoidal aparece un concepto denominado "Overranging" que se define como el aumento en el DLP del examen, producto de las rotaciones adicionales necesarias al comienzo y al fin de la exploración para poder realizar la reconstrucción del primer y último corte de la región explorada ${ }^{(22)}$. Esto es un factor inherente de la modalidad helicoidal, que siempre provocará una mayor extensión de barrido en comparación con la elegida por el operador en un principio, dando como resultado un aumento en el DLP entregado en el reporte dosimétrico de cada participante de la muestra estudiada, reflejando un aporte extra de dosis por efecto técnico.

A medida que aumenta la cobertura del barrido; y por ende la región explorada, aumenta la dosis entregada al paciente, traducido en el valor final del DLP. El aumento de la cobertura en los estudios se presenta con mayor frecuencia en los equipos de TC Multidetector, en los cuales generalmente no hay un aumento notorio del tiempo de barrido por aumentar la cobertura del examen, y en ocasiones se extiende un poco más allá de la estructura en estudio. Un ejemplo de lo anterior es el caso de los participantes estudiados con diagnóstico de TEC, los cuales por condición y afectación de partes blandas o huesos propios nasales podría determinar la extensión del barrido para incluir la totalidad de la zona. Si bien, podría reflejar un DLP más alto, los resultados obtenidos no reflejan diferencias estadísticas en los diagnósticos estudiados, por lo tanto, se debe considerar que siempre esta acción debe estar fundamentada respecto a la condición clínica del paciente y de una práctica responsable de parte del operador en función de la obtención de una imagen de utilidad diagnóstica.

El establecimiento de los valores típicos de dosis constituye un gran paso en el crecimiento de la Unidad de Radiología de la Institución, y como consecuencia una acción concreta que favorece directamente a los pacientes en cuanto a la protección y optimización de la dosis que deben recibir al ser estudiados a través de TC. De igual forma, se invita a repetir la experiencia en búsqueda de la disminución de los valores típicos de dosis, esto con la participación de los médicos a fin de que la calidad de imagen obtenida sea adecuada o suficiente para lograr el propósito clínico, repitiendo la experiencia cada 3 o 5 años, cuando se incorporen nuevas tecnologías, nuevos protocolos o exista un cambio en el post proceso, tal y como lo indican organizaciones de referencia ${ }^{(12)}$.

\section{Responsabilidades éticas}

Protección de personas y animales. Los autores declaran que para esta investigación no se han realizado experimentos en seres humanos ni animales.

Confidencialidad de los datos. Los autores declaran que en este artículo no aparecen datos de pacientes.

Derecho a la privacidad y consentimiento informado. Los autores declaran que en este artículo no aparecen datos de pacientes.

\section{Referencias}

1. UNSCEAR. Report to the General Assembly with Scientific Annexes. Sources and Effects of lonizing Radiation United Nations Scientific Committee on the Effects of Atomic Radiation, ANNEX A. 2010; 1: 25-30.

2. Costa Subias J, Soria Jerez JA. Principios Basicos. Tomografia Computarizada dirigida a Técnicos Superiores en Imagen para el Diagnóstico. Elsevier. 2015; 3-18.

3. Badel AE, Rico-Mesa JS, Gaviria MC, Arango-Isaza D, Hernández Chica CA. Radiación ionizante: revisión de tema y recomendaciones para la práctica. Rev Colomb Cardiol. 2018; 25(3): 222-229.

4. IAEA Radiation Protection of Patiens. Niveles de referencia para diagnóstico con tomografía computada (CT). Available from: https://rpop.iaea.org/RPOP/RPoP/ 
Content-es/InformationFor/HealthProfessionals/1_Radiology/ComputedTomography/diagnostic-referencelevels.htm\#IPEM2004

5. Ubeda C, Vaño E, Ruiz R, Soffia P, Fabri D. Niveles de referencia para diagnóstico: Una herramienta efectiva para la protección radiológica de pacientes. Rev Chil Radiol. 2019; 25(1): 19-25.

6. Vañó E, Miller DL, Martin CJ, Rehani MM, Kang K, Rosenstein M, et al. ICRP Publication 135: Diagnostic Reference Levels in Medical Imaging. Ann ICRP. 2017; 46(1): 1-144.

7. MINSAL. Sistema Reportes REM, Ministerio de Salud. REM-B17. Actividades de apoyo diagnostico y terapeutico. 2018 (cited 2019 nov 4). Available from: http://webdeis.minsal.cl/rem2018/?serie $=3 \&$ rem $=51 \&$ seccion_id $=510 \&$ tipo $=4 \&$ tipoReload $=3 \&$ regiones $=5 \&$ regiones $R$ eload $=0 \&$ servicios $=0 \&$ serviciosReload $=0 \&$ periodo $=2$ 018\&mes_inicio $=1 \&$ mes_final $=12$

8. MINSAL. Sistema Reportes REM 2018. REM-B17. Actividades de apoyo diagnóstico y terapeutico. Región Valparaiso. 2018 (cited 2019 nov 4). Available from: http:// webdeis. $\mathrm{minsal}$.cl/rem2018/?serie $=3 \& \mathrm{rem}=51 \&$ seccion $\mathrm{id}=510$ \&tipo $=4 \&$ tipoReload $=4$ \& regiones $=5$ \& regiones Reload $=5 \&$ servicios $=0 \&$ serviciosReload $=0$ \&periodo $=2018 \&$ mes_inicio $=1 \&$ mes_final $=12$

9. MINSAL. Sistema Reportes REM 2017. REM-B17. Actividades de apoyo diagnóstico y terapeutico. Region Valparaiso. 2017 (cited 2019 nov 4). Available from: http:// webdeis. $\mathrm{minsal}$.cl $/ \mathrm{rem} 2018 /$ ?serie $=3 \& \mathrm{rem}=46 \&$ seccion $\mathrm{id}=454 \&$ tipo $=4 \&$ tipoReload $=4 \&$ regiones $=5 \&$ regiones Reload $=5 \&$ servicios $=0 \&$ serviciosReload $=0$ \&periodo =2017\&mes_inicio=1\&mes_final $=12$

10. MINSAL. Sistema Reportes REM, Ministerio de Salud. REM-B17. Actividades de apoyo diagnostico y terapeutico. 2017 (cited 2019 nov 4). Available from: http://webdeis.minsal.cl/rem2018/?serie $=3 \&$ rem $=46 \&$ seccion_id $=454 \&$ tipo $=4 \&$ tipoReload $=4 \&$ regiones $=-1 \&$ regiones $R$ eload $=5 \&$ servicios $=0$ \&serviciosReload $=0$ \&periodo $=2$ $017 \&$ mes_inicio $=1 \&$ mes_final $=12$

11. MINSAL. Algunas actividades realizadas en establecimientos no pertenecientes al Sistema Nacional de Servicios de Salud, Imagenología. 2017 (cited 2019 nov 5). Available from: https://reportesdeis.minsal.cl/ remsas/2017/remsas01seccionh/remsas01seccionh. aspx
12. Rosenstein M. Diagnostic reference levels for medical exposure of patients: ICRP guidance and related ICRU quantities. Health Phys. 2008; 95(5): 528-534.

13. Biblioteca del Congreso Nacional de Chile. Norma T, Ley A, Bases S, Del G, Ambiente M (documento generado el 04-Dic-2013). 2013; Available from: www. leychile.cl/N?i=9794\& $\mathrm{f}=1984-08-23 \& p=$

14. Delgado O, Espinoza A, Edding O, Guerrero C, Martínez M. Trabajador(a) Ocupacionalmente Expuesto(a) a Radiaciones lonizantes. Nota técnica $N^{\circ} 34$ del Inst Salud Pública Chile. 2014; 7-9.

15. MINSAL. Aprueba Reglamento De Protección Radiológica. Subsecretaría De Salud Pública División Políticas Públicas Salu-Dables Y Promoción División Jurídica. 2016; Available from: https://www.minsal. cl/wp-content/uploads/2017/01/Consulta-públicaReglamento-de-Protección-Radiológica.pdf

16. I of PS in. National Protocol for Patient Dose Measurements in Diagnostic Radiology. Chilton. Didcot, Oxon: Dosimetry Working Party of the Institute of Physical Sciencies in Medicina; 1992: 1-46 p.

17. ImPACT CT Scanner Evaluation Group. Patient Dosimetry Calculator: 64 Slice Scanners. 2006. Available from: http://www.impactscan.org/download/ImPACT_CTDosimetry_Spreadsheet_64update.pdf

18. International Atomic Energy Agency. Quality Assurance for Computed Tomography - Diagnostic and Therapy Applications. Vienna; 2012: 19: 1-192.

19. Allende F, Lobos V, Zúñiga A. Corrección de índice de dosis en tomografía computarizada por parámetro de diámetro efectivo según reporte 204 de la Asociación Americana de Físicos Médicos. Rev Chil Radiol. 2016; 22(2): 61-69.

20. IEC. Evaluation and Routine Testing in Medical Imaging Departments: Part 2-6: Constancy Tests: X-ray Equipment for Computed Tomography. I.S. EN 61223-2-6: 2007. Ginebra, Suiza.

21. IEC. Evaluation and routine testing in medical imaging departaments-Part 3-5: Acceptance and constancy test - Imaging performance of computes tomography X-ray equipment. Edition 2.0. Ginebra, Suiza; 2019.

22. Kalender AW. "Computed Tomography. Fundamentals, System Technology, Image Quality, Applications”. Wiley \& Sons, Incorporated, John; 2000: 1-220 p. 